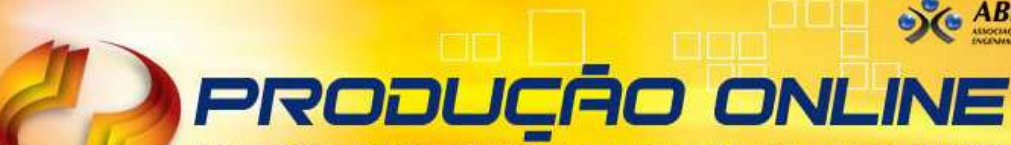 \\ REVISTA CIENTIFICA ELETRONNICA DE ENGENHARIA DE PRODUÇÃO \\ ISSN 1676-1901
}

\section{MODELO DE GERENCIAMENTO DA CAPACIDADE PRODUTIVA: INTEGRANDO TEORIA DAS RESTRIÇÕES E O ÍNDICE DE RENDIMENTO OPERACIONAL GLOBAL (IROG)}

\section{MANAGEMENT MODEL OF PRODUCTIVE CAPACITY: INTEGRATING THEORY OF CONSTRAINTS AND THE GLOBAL OPERATIONAL EFFICIENCY INDEX (IROG)}

\author{
Diego Augusto de Jesus Pacheco*E-mail: engdie@gmail.com \\ José Antonio Valle Antunes Júnior* E-mail: junico@unisinos.br \\ Daniel Pacheco Lacerda*E-mail: lacerda@unisinos.br \\ Dieter Brackmann Goldmeyer* E-mail: dieter.goldmeyer@gmail.com \\ Charles Von Gilsa* E-mail: cvongilsa@gmail.com \\ *UNISINOS - Universidade do Vale do Rio dos Sinos, São Leopoldo - RS
}

Resumo: Esse artigo apresenta um modelo de gerenciamento da capacidade produtiva em sistemas produtivos integrando os conceitos da Teoria das Restrições e da Total Productive Maintenance (TPM). O principal objetivo do trabalho é discutir e propor um modelo de gerenciamento da capacidade, capaz de responder às seguintes questões centrais: i) quais indicadores de capacidade devem ser considerados e como medi-los para mensurar a capacidade produtiva de sistemas de manufatura? ii) qual é a real capacidade do sistema produtivo analisado frente a uma determinada relação entre capacidade e demanda? A discussão do modelo proposto é relevante na medida em que a definição da capacidade produtiva de sistemas possibilita o melhor gerenciamento dos recursos e de suas capacidades, melhora a programação da produção no chão de fábrica e 0 atendimento às demandas impostas pelo mercado. Esse artigo apresenta a proposição do uso do índice do Rendimento Operacional Global (IROG) com uma abordagem diferente da que consta na literatura tradicional que trata do tema, apresentada por Nakajima (1988). Os resultados desse artigo permitiram desenvolver um modelo capaz de determinar a capacidade do sistema produtivo e o impacto sobre a capacidade produtiva de todo o sistema, ao se considerar as não conformidades de qualidade que ocorrem após o recurso gargalo do fluxo produtivo.

Palavras-chave: Gerenciamento de capacidade. Teoria das Restrições. Total Productive Maintenance.

Abstract: This paper presents a model of management capacity in productive systems integrating the concepts of the Theory of Constraints and Total Productive Maintenance (TPM). The main objective of this study is to discuss and propose a model of management capacity, able to answer the following key questions: i) capacity indicators which should be considered and how to measure them to measure the productive capacity of manufacturing systems? ii) what is the real productive capacity of the system analyzed under a determined relationship between capacity and demand? The discussion of the proposed model is relevant because the definition of productive capacity system enables better management of resources and capabilities, improve production scheduling on the factory floor and meeting the demands imposed by the market. This paper presents the proposition of using the Operating Income Index Global (IROG) with a different approach from traditional literature dealing with the theme, presented by Nakajima (1988). The results of this paper enable to develop a model to determine the capacity of the production system and the impact on the productive capacity of the 
entire system, not to consider the quality conformances that occur after the bottleneck resource of the production flow.

Key-words: Capacity management. Theory of Constraints. Total Productive Maintenance.

\section{INTRODUÇÃO}

Para Nakajima (1988) o indicador de Índice do Rendimento Operacional Global (IROG) deve ser considerado como um indicador operacional e pode ser aplicado em diversos níveis dentro de um sistema de manufatura. Num primeiro momento o IROG pode ser usado com benchmarking para medir o nível de desempenho de um sistema produtivo de forma global. Ou seja, o IROG é medido inicialmente e comparado com um IROG futuro após o sistema ter passado por um programa de melhorias ou então comparado com o desempenho de outros sistemas semelhantes. O IROG também pode ser calculado em sistemas de manufatura com diversas linhas de produção, proporcionando verificar quais os reais níveis de utilização dos ativos da indústria (MUTHIAH e HUANG, 2007; MUTHIAH et al., 2008). Outra perspectiva de adoção do cálculo do IROG é no contexto de equipamentos individuais, resultando em uma métrica quantitativa de produtividade e possibilitando assim identificar quais máquinas possuem desempenho alto ou baixo (em relação a uma dada meta), se definindo assim como um relevante aspecto do TPM (BRAGLIA et al., 2008; MATHUR et al., 2011; MUTHIAH e HUANG, 2007). De forma geral, esse indicador é importante porque permite mensurar a real capacidade produtiva dos equipamentos para um período de tempo pré-determinado ou demanda conhecida. Nesse sentido, torna-se relevante explorar o conceito do IROG para a discussão da capacidade tanto de equipamentos, de forma específica, como de sistemas produtivos complexos, de forma mais ampla.

Já a Teoria das Restrições criada por Goldratt (1984) propõe uma metodologia para identificar a restrição de capacidade que define o desempenho global do sistema produtivo e, a partir daí, explorá-la a fim de elevar a capacidade instalada e o ganho global. A restrição de capacidade é o gargalo do sistema, que é definido como sendo o recurso cuja capacidade disponível é menor do que a capacidade necessária para atender a demanda imposta a ele, num determinado período de tempo, o qual é geralmente longo (ANTUNES, 1998). O método Tambor- 
Pulmão-Corda é o algoritmo usado para explorar a capacidade do gargalo e será detalhado nesse estudo.

Assim sendo, esse artigo apresenta um modelo de gerenciamento da capacidade produtiva em sistemas produtivos integrando os conceitos da Teoria das Restrições e da Total Productive Maintenance (TPM). O objetivo do presente trabalho é discutir e sugerir um modelo de gerenciamento da capacidade, capaz de responder às seguintes questões: quais indicadores de capacidade devem ser considerados e como medi-los para mensurar a capacidade de sistemas de manufatura? ii) qual o real capacidade do sistema produtivo em análise frente a uma determinada relação entre capacidade e demanda. Para atender a tal propósito esse artigo esta estruturado da seguinte maneira: as seções 2, 3 e 4 apresentam objetivamente a revisão da literatura sobre capacidade produtiva, TPM, IROG e Teoria das Restrições; a seção 5 apresenta e descreve as etapas do modelo proposto; a seção 6 apresenta a aplicação teórica e a discussão sobre o modelo; e a seção 7 descreve as conclusões e as sugestões de trabalhos futuros.

\section{CAPACIDADE PRODUTIVA INDUSTRIAL}

Hayes et al. (2008) argumentam que medir a capacidade de sistemas produtivos é uma tarefa complexa, devido à ação dos seguintes fatores associados à variabilidade: políticas da empresa, confiabilidade dos fornecedores,confiabilidade dos equipamentos, taxas de produção e o impacto dos fatores humanos. Para Hopp e Spearman (2001) a variabilidade existe em todos os sistemas de produção e pode causar um grande impacto no seu desempenho. Por esta razão, a habilidade de medir, compreender e gerenciá-la torna-se crítica para uma administração eficaz da produção.

Hopp e Spearman (2001) também apresentaram os seguintes pressupostos sobre o gerenciamento da capacidade produtiva em sistemas: i) uma linha de fluxo desequilibrada com um gargalo conhecido é mais fácil de administrar e demonstra um comportamento logístico melhor quando comparado com uma linha em equilíbrio, onde os tempos de processamento das operações são semelhantes; ii) a capacidade está geralmente disponível apenas em tamanhos incrementais prédeterminados,ou seja, podemos comprar um ou dois recursos, mas não um e meio e 
pode ser impossível ajustar a capacidade de determinada operação com a meta de produção; iii) o custo da capacidade, geralmente, não é o mesmo em cada estação de trabalho e é mais barato manter capacidade em excesso em algumas estações do que em outras.

Avançando na discussão sobre capacidade, segundo Antunes et al. (2008) a capacidade e a demanda de um dado recurso, em unidades de tempo, podem ser determinadas respectivamente, de acordo com as equações 1 e 2 :

$$
C=T_{t} \times \mu_{g}
$$

Onde:

C: Capacidade de produção para a produção; Tt: Tempo total disponível para a produção (tempo); $\mu \mathrm{g}$ : Índice de Rendimento Operacional Global do equipamento (IROG). Já a demanda, em unidades de tempo de um dado recurso pode ser calculada de acordo com o que determina a equação 2 .

$$
D=\sum_{i=1}^{N} t p_{i} \times q_{i}
$$

Onde:

D: Demanda de produtos no equipamento (tempo); Tpi: Taxa de processamento da peça i no equipamento (tempo por unidade de produção); qi: Quantidade produzida da peça i no equipamento (unidades de produção).

\section{TOTAL PRODUCTIVE MAINTENANCE (TPM) E O IROG}

$O$ conceito de eficiência dos equipamentos e o termo IROG foram apresentados por Nakajima (1988). Sua discussão é central para o cálculo da capacidade porque determina, a priori, a capacidade prática e não teórica dos equipamentos e sistemas. A equação 3 apresenta a equação genérica de cálculo do IROG:

$$
\mu_{\text {global }}=\frac{\sum_{i=1}^{n} t p_{i} x q_{i}}{T}
$$

Onde: 
$\mu \mathrm{g}$ : Índice de Rendimento Operacional Global do equipamento (IROG); tp: é o tempo de ciclo ou tempo padrão do produto $X$; $q$ : é a quantidade de produtos $X$ processados; T: é o tempo disponível para a produção.

Verifica-se nesta equação, que a multiplicação do tempo de ciclo de um produto pela quantidade produzida deste produto em um determinado equipamento corresponde ao tempo de agregação de valor deste equipamento no processo de produção sobre o prisma das práticas enxutas (ANTUNES, 1998). Ou seja, trata-se do tempo em que o equipamento efetivamente o mesmo funcionou adicionando valor ao produto. O tempo de ciclo é o tempo que um operador devidamente treinado processa uma peça em um equipamento específico, de forma a realizá-la normalmente. Isto é, sem realizar esta atividade velozmente, de maneira a não conseguir manter o mesmo ritmo durante o seu período de trabalho, e sem realizá-la de forma muito lenta, como ocorre normalmente quando um operador se encontra em fase de treinamento ou não está familiarizado com a operação. O IROG que vem sendo usado e bem aceito por várias empresas de diferentes setores industriais a partir de Nakajima (1988). Apesar se ser considerado um medidor ótimo, indica qual é a direção para a realização de melhorias no equipamento ou sistema analisado (BRAGLIA et al., 2008; MATHUR et al., 2001). O cálculo do IROG é feito considerando os seguintes aspectos:

a) Se o posto de trabalho é um recurso gargalo: neste caso, o indicador IROG é denominado de TEEP, ou seja, Produtividade Efetiva Total do Equipamento (Total Effective Equipment Productivity). Nesse caso, o tempo T considerado na equação 3 é o tempo total - no caso dos recursos críticos gargalos, 24 horas/dia (MATHUR et al., 2011). Isto se explica pelo fato de que, sendo o posto de trabalho um gargalo, todo o tempo disponível deve ser utilizado na produção. Este índice indica o tempo que pode ser ganho para produzir e corresponde a produtividade real do sistema produtivo a partir do gargalo.

b) Se o posto de trabalho é um recurso não gargalo: neste caso, o indicador IROG é denominado de OEE, ou seja, Índice de Eficiência Global (Overall Equipment Efficiency). $O$ tempo $T$ considerado na equação 3 é o tempo 
disponível, obtido pela diferença entre o tempo total e o tempo das paradas programadas. Por não se tratar de um posto de trabalho gargalo, é possível programar certas paradas como: parada para almoço, ginástica laboral, etc., uma vez que a não paralisação deste equipamento geraria estoques intermediários antes do gargalo. Este índice indica a eficácia do equipamento durante o tempo de operação programado.

As considerações acima consistem no cálculo da eficiência global dos equipamentos través da equação geral, devendo ser desdobradas, segundo Nakajima (1988) com a finalidade de identificar as principais causas das ineficiências observadas no posto de trabalho. O cálculo da eficiência global é então definido pelos seguintes índices de eficiência, conforme Equação 4 e descritos a seguir:

$$
\mu_{\text {global }}=\mu_{1} x \mu_{2} x \mu_{3}
$$

Onde:

$\mu 1$ : Índice de Tempo Operacional - ITO;

2: Índice de Performance Operacional - IPO;

33: Índice de Produtos Aprovados - IPA.

O ITO corresponde ao tempo em que o equipamento ficou disponível, excluindo-se as paradas não programadas. É relacionado, portanto, com a paralisação do equipamento. Isto é, quando a velocidade do mesmo cai para zero como quebras, trocas de ferramentas e ajustes (MATHUR et al., 2011; MUTHIAH e HUANG, 2007). Quanto menor for o valor do ITO, maior será o potencial de aumento da utilização do posto de trabalho. Isso ocorre porque um baixo valor indica que o equipamento sofre muitas paradas não programadas. $E$ os recursos que processam pouca variedade de peças, tendem a ter um ITO elevado devido a baixa necessidade de trocas de ferramentas, preparações e ajustes.

O IPO mede o desempenho operacional do recurso, sendo calculado em função do tempo disponível e à redução da velocidade do mesmo, operação em vazio e paradas momentâneas (MUTHIAH e HUANG, 2007). É relacionado, portanto, com a queda de velocidade do recurso (velocidade diferente da nominal e diferente de zero). As causas que geram valor baixo do IPO podem ser: i) do tipo técnicas, como operações em vazio por falta de alimentação de peças (não havendo assim agregação de valor) ou tempo de ciclo alto com a conseqüente redução da 
velocidade do processamento; ii) de rotina, como o não registro dos dados no diário de bordo ou sistema de coleta (por exemplo, paradas pequenas e sucessivas, onde o somatório tem impacto significativo no valor final do IPO). O último indicador analisado é o IPA que mede a qualidade das peças produzidas, sendo calculado em função do tempo de operação real, excluindo-se o tempo gasto com refugos ou retrabalhos (MATHUR et al., 2011; MUTHIAH et al., 2008).

A literatura evidencia que após o surgimento do IROG a partir da obra de Nakajima (1988), surgiram outros medidores, os quais derivam essencialmente do IROG. Nesse caso, é relevante para esse trabalho citar o OFE (Overall Factory Effectiveness) e o OTE (Overall Tthroughput Eeffectiveness), que foram elaborados para avaliar o desempenho de sistemas ou subsistemas inteiros (MUTHIAH et al., 2008; MUTHIAH e HUANG, 2007; MATHUR et al., 2011). Uma perspectiva mais ampla de implantação e desdobramentos de tais indicadores pode ser encontrada em Scott e Pisa (1998), Muthiah et al. (2008) e Muthiah e Huang (2007).

É importante também salientar que a discussão integrando TPM, IROG e a TOC, foi sugerida e analisada na literatura por alguns autores (JEONG e PHILLIPS, 2001; ROSE et al.,1995; CHAKRAVORTY e ATWATER, 2006). A discussão proposta por tais autores corrobora com os esforços de pesquisa do modelo alternativo proposto no presente trabalho. Conforme Jeong e Phillips (2001), por exemplo, a TPM enfatiza a manutenção autônoma e os recursos gargalos deveriam ter maior prioridade em detrimento dos recursos não gargalos dos sistemas produtivos. $O$ IROG, segundo Rose et al. (1995), quando aplicado ao recurso gargalo, pode aumentar o ganho do sistema. Sendo assim, de forma geral pode-se concluir que o gerenciamento de gargalos sugerido pela TOC, somando-se ao indicador IROG oriundo da abordagem da TPM, podem contribuir não só para efetivo aumento da produtividade, como também para elevar o ganho do sistema.

\section{TEORIA DAS RESTRIÇÕES}

Segundo Cox e Spencer (2002), a Teoria das Restrições pode ser entendida a partir dos seus seguintes componentes: 
a) Uma abordagem Logística e de Operações, que envolve os seguintes métodos: i) Os cinco passos envolvendo o foco na melhoria dos processos; ii) O processo de programação da produção envolvendo o gerenciamento via a lógica TPC (Tambor, Pulmão e Corda) e o gerenciamento dos pulmões no sistema produtivo; iii) A análise dos sistemas produtivos adotando a classificação V-A-T.

b) A proposição de um Sistema de Indicadores de Desempenho, que passa pela: i) Definição dos Ganhos, Inventários e Despesas Operacionais da Empresa; ii) Definição do mix de produtos que deverá ser produzido visando maximizar os resultados; iii) A lógica dos Ganhos por dia e dos Inventários por dia.

c) E por fim, a TOC pode ser entendida como um Processo de Pensamento visando à solução de problemas, que envolve as seguintes técnicas: i) Os diagramas de efeito-causa-efeito, que são: a Árvore da Realidade Atual, Árvore da Realidade Futura, Árvore dos Pré-Requisitos e Árvore de Transição; ii) O método da Evaporação das Nuvens.

Os cinco passos de melhoria contínua dos processos da TOC podem ser aplicados para o gerenciamento do gargalo do sistema. Por isso o detalhamento desse método é relevante na discussão dessa pesquisa sobre gerenciamento da capacidade produtiva. De forma objetiva, os cinco passos da TOC para melhoria de processos segundo Goldratt (1996) são:

1. Identificar a(s) restrição(ões) do sistema: elas podem ser internas ou externas à empresa. Quando a demanda total de um dado mix de produtos é maior do que a capacidade da fábrica se diz que existe um gargalo de produção. Todavia, quando a capacidade de produção é superior à demanda de produção, a restrição é externa ao sistema.

2. Explorar da melhor forma possível a(s) restrição(ões) do sistema: se a restrição é interna à fábrica, a melhor decisão consiste em maximizar o ganho no(s) gargalo(s), fazendo que o maior número possível de produtos prédefinidos pelo critério de maior geração de ganho, passem pelo gargalo.

3. Subordinar todos os demais recursos à decisão tomada no passo 2: a lógica deste passo, independentemente da restrição ser externa ou interna, 
consiste em reduzir ao máximo os inventários e as despesas operacionais e ao mesmo tempo garantir o ganho teórico máximo do sistema de produção.

4. Elevar a capacidade da(s) restrição(ões): se o gargalo for interno é necessário aumentar sua capacidade produtiva. Isso pode ser feito através de mudanças de leiaute, compra de equipamentos, uso de horas extras, redução da variabilidade, redução de setup, aplicação das técnicas da manufatura enxuta, etc.

5. Voltar ao passo 1 para não deixar que a inércia tome conta do sistema: ao elevar a capacidade produtiva da restrição o sistema torna-se, a priori, um sistema genérico, o que gera a necessidade de analisá-lo novamente. $O$ passo cinco retoma a idéia de melhoria contínua dos processos.

\subsection{O algoritmo Tambor-Pulmão-Corda (TPC) e o aumento da capacidade}

Para Goldratt (1996) o TPC visa operacionalizar no chão de fábrica os cinco passos de melhoria dos processos da TOC. A lógica central do algoritmo TPC consiste em estabelecer a produção puxada para controlar o fluxo de inventário no sistema produtivo (WATSON e PATTY, 2009). Tanto os cinco passos de melhoria de processos, já supracitados, quanto o TPC, estão inseridos no modelo discutido nesse trabalho. Assim sendo, de forma objetiva os elementos da lógica TPC serão descritos a seguir, e estão representados na Figura 1:

a) Tambor (T): é o gargalo do sistema, o qual determina sua capacidade produtiva total (WATSON e PATTY, 2009; UMBLE e UMBLE, 1999). Portanto, define o ritmo da produção e restringe a capacidade, ou seja, é o Tambor do sistema, uma vez que dita o seu ritmo de produção (DETTMER, 2001; RAHMAN, 1998).

b) Pulmão (P): é a proteção colocada antes do tambor para evitar interrupções no tambor devido ao impacto das variabilidades, como quebra de máquinas, variação no tempo de processo, problemas de qualidade, falta de matéria prima para produzir, etc. Em outras palavras, pulmão é um elemento estratégico que busca proteger o ganho do sistema sobre as variações do mesmo (WATSON e PATTY, 2009; RAHMAN, 1998). Há três tipos de pulmão 
que podem ser usados nesse caso: pulmão de tempo, inventário ou de capacidade.

c) Corda (C): tem o objetivo de sinalizar a necessidade de entrada de materiais no sistema, para alimentar o pulmão e o gargalo, limitando a quantidade de matéria-prima liberada para a fábrica. Assim sendo, a função da corda, que pode ser um fluxo físico ou de informação, é liberar os materiais em função do consumo de materiais do gargalo (WATSON e PATTY, 2009).

Figura 1 - Algoritmo TPC

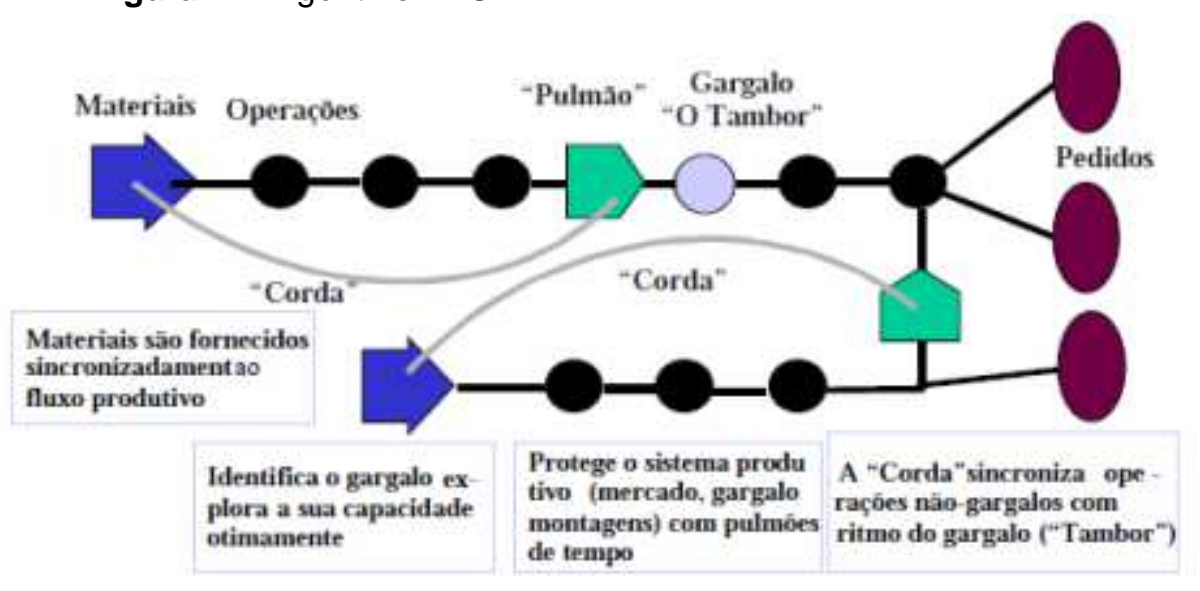

Fonte: Antunes (1998).

O algoritmo TPC sincroniza a utilização dos recursos e materiais, os quais são usados apenas em um determinado nível para a geração de ganho no processo (UMBLE e UMBLE, 1999; RAHMAN, 1998). As vantagens de uso do TPC são corroboradas com a evidência de resultados de implantações presentes na literatura (GOLDRATT, 2009). A pesquisa de Mabin e Baderstorne (2003) evidenciou estes benefícios a partir da melhoria de desempenho no lead time, nos tempo de ciclo e na ampliação das receitas, ou ganho na lógica da TOC, de organizações que aplicaram o TPC. Concordando com estas evidências, Patty e Watson (2008) concluíram que empresas que utilizam TPC alcançam maiores ganhos gerando menos estoques. Sendo assim, o TPC é um algoritmo que objetiva, por meio dos cinco passos de focalização da TOC coordenar sistemas produtivos (GUPTA e BOYD, 2008). E Ifandoudas e Chapman (2009) afirmam que este processo busca determinar o que, quando e como a produção será coordenada de forma a reduzir inventário e maximizar as vendas. 


\section{DESCRIÇÃO DO MODELO DE GERENCIAMENTO DA CAPACIDADE PRODUTIVA}

O modelo proposto nessa pesquisa possui os seguintes pressupostos considerados no desenvolvimento do método os quais devem ser avaliados na sua aplicação empírica. Em primeiro lugar, o modelo considera que a restrição da empresa é interna ao sistema produtivo, podendo ser um recurso físico, equipamento, linha de produção, célula, etc. Outro pressuposto do modelo é que a variedade de produtos fabricados em qualquer recurso do sistema não impacta nos resultados apresentados. Ou seja, o método pode ser aplicado em ambientes de manufatura de alto ou baixo nível de customização dos produtos fabricados.

O modelo também se adapta tanto para sistemas de manufatura de produção puxada ou quanto para empurrada. Um aspecto positivo do modelo é que o tamanho do período de tempo em análise não influencia no modelo. Pode-se analisar um dia, um mês, um ano e assim por diante. Um aspecto fundamental a considerar antes de se aplicar o método é que as informações de entrada como: quantidades demandadas e principalmente os tempos de processamento sejam confiáveis. Esse aspecto é um pré-requisito para se definir com precisão qual recurso é a restrição do sistema. Será analisado um caso teórico em uma linha de produção composta de cinco recursos onde são manufaturados um tipo de produto, denominado de $X$, composto de dois componentes ( $\mathrm{A}$ e B). $\mathrm{O}$ modelo proposto é composto pelas seguintes etapas, conforme Figura 2: 
Figura 2 - Etapas do modelo de gerenciamento da capacidade produtiva integrando TOC e TPM

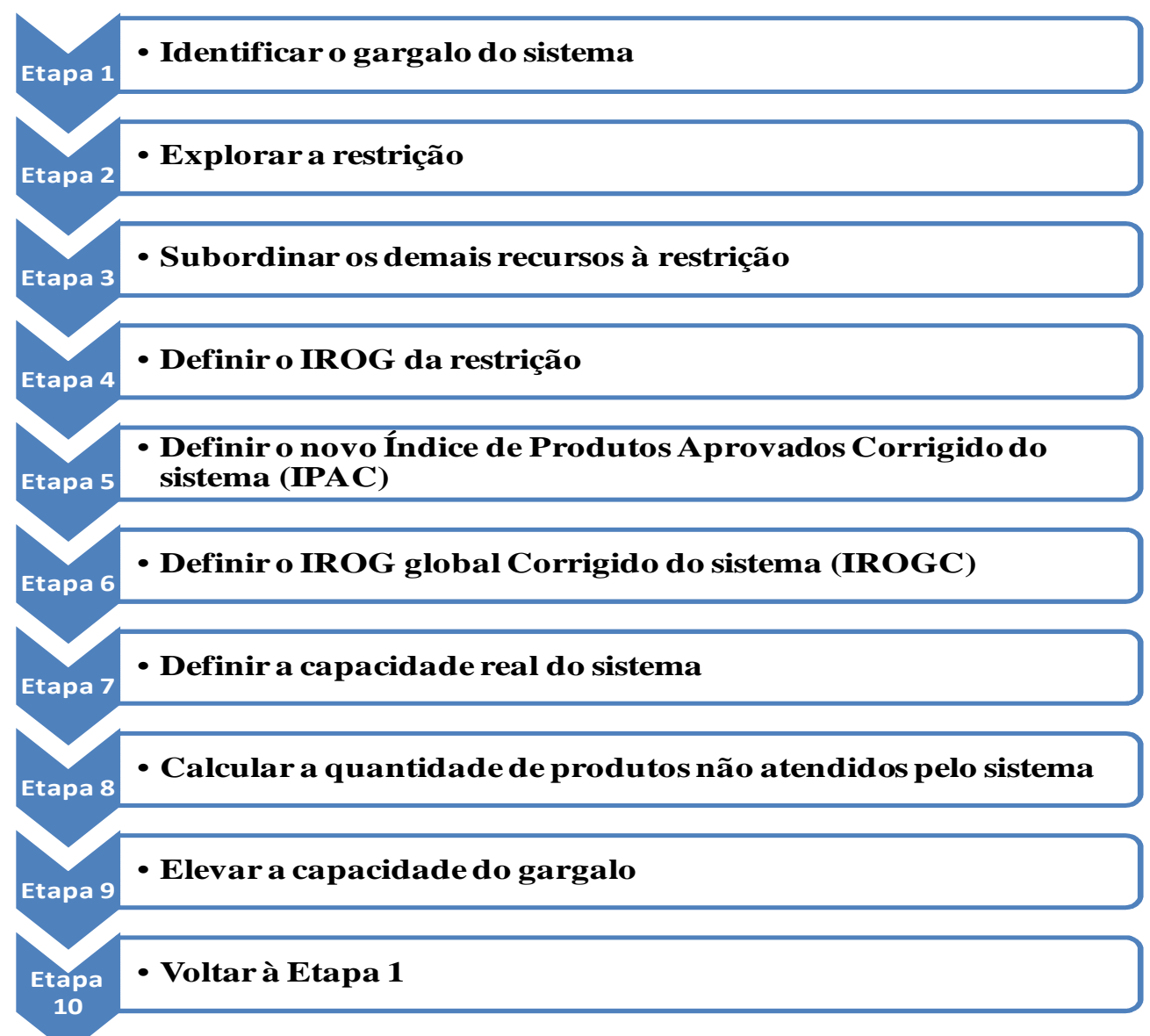

Fonte: Elaborado pelos autores (2012).

A seguir, serão apresentadas e descritas as etapas do modelo proposto no presente estudo.

1. Identificar a restrição do sistema: nessa etapa do modelo é identificada a restrição do sistema, cuja capacidade é inferior à demanda imposta, a partir das análises do passo 1 da TOC. Nesse caso, a análise baseou-se na abordagem de Capacidade x Demanda proposta por Antunes et al. (2008) e fez a proposição de algumas alterações nesse método de análise dos autores. As alterações sugeridas de forma e ampliar o modelo de Capacidade $x$ Demanda de Antunes et al. (2008) está apresentado na Tabela 2.

2. Explorar a restrição: nessa etapa o gargalo do sistema é explorado visando maximizar o ganho do sistema, porém sem investimentos adicionais, conforme preconiza o passo 2 do processo de focalização da TOC. 
3. Subordinar os demais recursos à restrição: nessa etapa os recursos que estão dispostos no leiaute do sistema produtivo, tanto à jusante quanto à montante do gargalo, são subordinados ao gargalo, de acordo com o passo 3 da TOC. O objetivo dessa etapa do modelo é assegurar que a capacidade do gargalo irá definir o planejamento da produção no sistema produtivo.

4. Definir o IROG (Índice de Rendimento Operacional Global) da restrição: nessa etapa do modelo calcula-se o TEEP do gargalo conforme apresentado e discutido previamente na seção 3. O objetivo principal dessa etapa é avaliar o nível atual de desempenho do recurso gargalo do sistema.

5. Definir o novo Índice de Produtos Aprovados Corrigido (IPAC) do sistema: nessa etapa do modelo, calcula-se o IPA de todos os recursos dispostos no fluxo de produção após o gargalo e efetua-se a multiplicação entre esses índices calculados, obtendo-se assim num valor único, denominado de IPA Corrigido (IPAC). Ao mensurar a quantidade de produtos rejeitados no fluxo após o gargalo, é possível medir o real índice de produtos conformes que são produzidos após o gargalo contribuindo para um dimensionamento mais acurado da capacidade global do sistema. Esse aspecto do modelo, é uma das principais lacunas que o presente estudo pretende preencher dentre os modelos de gerenciamento de capacidade.

6. Definir o IROG Corrigido do sistema (Índice de Rendimento Operacional Global Corrigido): nessa etapa do modelo é obtido o novo IROG do sistema, a partir da visão de capacidade dos gargalos da TOC, denominado nesse estudo de IROGC. O resultado é obtido multiplicando-se o IROG calculado na etapa 4 pelo indicador IPAC obtido na etapa 5.

7. Definir a capacidade real do sistema: nessa etapa do modelo define-se a capacidade real do sistema em atender à demanda a partir da capacidade do gargalo. O resultado é obtido multiplicando-se o tempo disponível para a produção no recurso gargalo pelo valor do IROGC obtido na etapa 6. Dessa maneira, a partir da análise da diferença temporal resultante da análise, é possível avaliar se gargalo do sistema possui capacidade de atender à demanda imposta analisada. 


\section{Calcular a quantidade teórica de produtos não atendidos pelo sistema:}

essa etapa do modelo consiste em dividir a diferença temporal do gargalo pelo somatório dos tempos de processamento do produto no recurso gargalo. Para fins de tomada de decisão gerencial, sugere-se que o resultado obtido seja arredondado para cima para minimizar o efeito de adição incremental da capacidade, conforme visto em Hopp e Spearman (2001) e Umble e Srikanth (1995). O valor então obtido é a quantidade de produtos não atendida pelo sistema. O resultado é dito como teórico porque está relacionado diretamente com a confiabilidade dos dados de entrada de cálculo nos passos anteriores do modelo. Logo, nessa etapa do modelo se evidencia a importância de se ter uma base de dados confiável para as análises dos resultados apresentados pelo modelo. Além disso, se define como uma quantidade teórica porque é praticamente inviável prever com precisão o comportamento futuro de um sistema devido ao efeito das variabilidades dos processos produtivos (HOPP e SPEARMAN, 2001). Assim sendo, caso depois de realizadas as etapas anteriores, seja verificado que há recursos com diferença temporal negativa, deve-se calcular novamente a quantidade teórica de produtos não atendida. Nesse caso, a quantidade de produtos não entregues pelo sistema, será o somatório dos produtos não entregues em todos os equipamentos com diferença temporal negativa.

9. Elevar a capacidade do gargalo: nessa etapa são realizadas ações, caso sejam necessárias, para elevar a capacidade do gargalo de acordo com o passo 4 da TOC. Nesse ínterim, diversas estratégias gerenciais podem ser adotadas, tais como: aplicar as técnicas enxutas de redução de perdas, reduzindo a variabilidade, reduzindo os tempos de setup, adquirindo outros recursos, usando horas extras, contratação de novos trabalhadores, usar roteiros alternativos reduzindo o tempo de operação dos produtos no gargalo, e assim por diante.

10. Voltar à etapa 1: a lógica dessa etapa do modelo consiste em reavaliá-lo, aplicado o sentido de melhoria contínua de processos. Pretende-se nessa etapa, à luz da TOC, evitar que a inércia tome conta do sistema produtivo, de acordo como passo 5 da TOC. 


\section{APLICAÇÃO TEÓRICA E DISCUSSÃO DO MODELO}

A etapa 1 do modelo consiste na etapa 1 dos 5 passos da TOC. Para identificar o gargalo do sistema usou-se inicialmente a relação entre capacidade e demanda proposta por Antunes et al. (2008), apresentada na Tabela 1, com o período de 1 mês de produção do produto em análise no modelo. Multiplicando-se a demanda mensal pelos tempos de processamento em cada equipamento, obteve-se a demanda mensal em unidade de tempo para cada equipamento. A demanda mensal total é o somatório das demandas dos componentes $\mathrm{A}$ e $\mathrm{B}$ em cada equipamento. O IROG de cada equipamento foi inicialmente calculado e depois multiplicado pelo tempo disponível em cada recurso para se obter a capacidade real em unidade de tempo por recurso. Por fim, fez-se a análise dos recursos onde constatou-se que, o recurso 2 não poderá atender à demanda de produtos imposta pelo mercado (-97) e é, portanto, a restrição do sistema.

Tabela 1 - Análise de capacidade versus demanda

\begin{tabular}{|c|c|c|c|c|c|c|c|c|c|c|c|}
\hline \multirow{2}{*}{ Produto } & \multirow{2}{*}{$\begin{array}{c}\text { Programação } \\
\text { Mensal }\end{array}$} & \multicolumn{5}{|c|}{ Tempo de ciclo por recurso $(h)$} & \multicolumn{5}{|c|}{ Demanda mensal por recurso $(\mathrm{h})$} \\
\hline & & Recurso 1 & Recurso 2 & Recurso 3 & Recurso 4 & Recurso 5 & Recurso 1 & Recurso 2 & Recurso 3 & Recurso 4 & Recurso 5 \\
\hline$A$ & \multirow{2}{*}{50} & 1 & 3 & 2.5 & 1 & 1 & 50 & 150 & 125 & 50 & 50 \\
\hline $\mathrm{B}$ & & 2 & 4 & 1 & 1 & 2 & 100 & 200 & 50 & 50 & 100 \\
\hline \multicolumn{7}{|c|}{ Demanda total por recurso (D) } & 150 & 350 & 175 & 100 & 150 \\
\hline \multicolumn{7}{|c|}{ Coeficiente do IROG- ITO } & 0.83 & 0.73 & 0.64 & 0.76 & 0.44 \\
\hline \multicolumn{7}{|c|}{ Coeficiente do IROG- IPO } & 0.64 & 0.78 & 0.59 & 0.47 & 0.82 \\
\hline \multicolumn{7}{|c|}{ Coeficiente do IROG- IPA } & 0.83 & 0.89 & 0.98 & 0.92 & 0.89 \\
\hline \multicolumn{7}{|c|}{ Índice de Rendimento Operacional Global (IROG) } & 0.44 & 0.51 & 0.37 & 0.33 & 0.32 \\
\hline \multicolumn{7}{|c|}{ Capacidade nominal do recurso (C) } & 500 & 500 & 500 & 500 & 500 \\
\hline \multicolumn{7}{|c|}{ Capacidade Real do recurso (C x IROG) } & 220 & 253 & 185 & 164 & 161 \\
\hline \multicolumn{7}{|c|}{ Diferença temporal $((\mathrm{C} \times \mathrm{IROG})-\mathrm{D})$} & 70 & -97 & 10 & 64 & 11 \\
\hline
\end{tabular}

Fonte: Elaborado pelos autores (2012).

As etapas 2 e 3 do modelo correspondem às respectivas etapas dos 5 passos de focalização da TOC. Para operacionalizar a aplicação dessas duas etapas será aplicado o algoritmo TPC da TOC. Primeiro o pulmão de proteção foi colocado na frente do recurso 2 que é o gargalo do sistema (Tambor). A corda irá sinalizar a necessidade de entrada de materiais no fluxo, para alimentar o pulmão e o gargalo, limitando a quantidade de matéria-prima liberada de acordo com a capacidade do gargalo, conforme Figura 3. Os dados de análise para realizar a etapa 4 já devem estar coletados na primeira etapa do modelo. Verifica-se nesse caso que o IROG da 
operação gargalo é de 0.51. Uma análise necessária a se fazer nesse momento é que, apesar das operações 3, 4 e 5 possuírem IROG menor que a operação 2, nenhuma das três é o gargalo. Esse aspecto ilustra a complexidade que envolve as análises sobre o tema da capacidade em sistemas de manufatura.

Figura 3 - Etapas 2 e 3 do modelo de aplicação do TPC

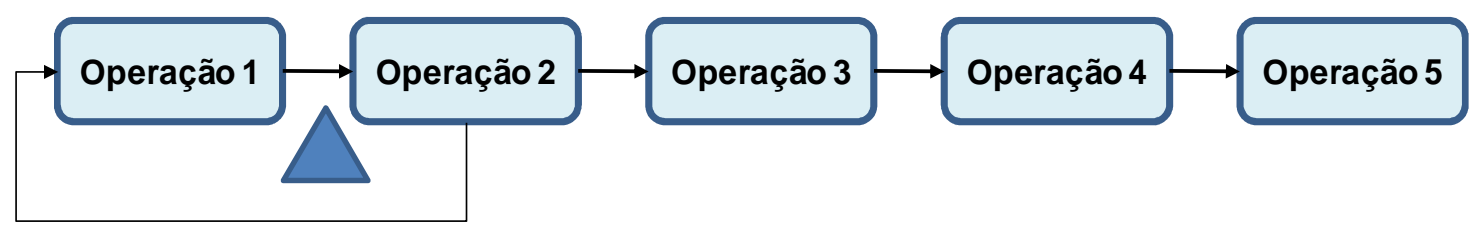

Fonte: elaborado pelos autores (2012).

O cálculo do IPAC de acordo com a etapa 5 é realizado multiplicando-se os valores dos coeficientes de qualidade (IPA) individuais de todos os recursos dispostos no leiaute após o gargalo (nesse caso os recursos 3, 4 e 5). Nesse caso, obteve-se o seguinte resultado: $0.98 \times 0.92 \times 0.89=0.80$. $O$ valor 0.80 encontrado indica a taxa de produtos conformes que são produzidos após o gargalo. Ao compará-lo com a demanda de produção (nesse caso 50 produtos), é possível então definir a quantidade de produtos aprovados produzidos no sistema. Para calcular o IROG Corrigido da etapa 6, multiplicou-se o IROG do gargalo (0.51) calculado na etapa 4 pelo valor do IPAC (0.8) da etapa 5 . Nesse caso $0.51 \times 0.8=0,41$. Conseqüentemente, a capacidade real do sistema também foi alterada. Assim sendo, ao se calcular a nova capacidade, conforme a etapa 7 do modelo, evidenciou-se que a capacidade do gargalo que antes era de 253 unidades de tempo, se reduziu para 203 unidades de tempo. Por conseguinte, a diferença temporal para cumprir a demanda mensal foi alterada de -97 unidades de tempo para -143 unidades de tempo, proporcionalmente à capacidade. O cálculo dos produtos não atendidos pelo sistema produtivo, conforme a etapa 8 foi realizado dividindo a nova diferença temporal (-143) pela soma dos tempos de processamento no recurso gargalo $(3+4=7 \mathrm{~h})$, resultando em 21 produtos. A análise dos resultados evidencia que na situação anterior, os resultados permitiram concluir que a quantidade de produtos não atendida pelo sistema era de 14, ou seja, uma diferença 
de $33.33 \%$. Os resultados da aplicação das etapas referidas estão apresentados na Tabela 2.

Tabela 2 - Cálculo da nova capacidade do sistema

\begin{tabular}{|c|c|c|c|c|c|c|c|c|c|c|c|}
\hline \multirow{2}{*}{ Produto } & \multirow{2}{*}{$\begin{array}{c}\text { Programação } \\
\text { Mensal }\end{array}$} & \multicolumn{5}{|c|}{ Tempo de ciclo por recurso (h) } & \multicolumn{5}{|c|}{ Demanda mensal por recurso (h) } \\
\hline & & Recurso 1 & Recurso 2 & Recurso 3 & Recurso 4 & Recurso 5 & Recurso1 & Recurso 2 & Recurso 3 & Recurso 4 & Recurso 5 \\
\hline A & \multirow{2}{*}{50} & 1 & 3 & 2.5 & 1 & 1 & 50 & 150 & 125 & 50 & 50 \\
\hline $\mathrm{B}$ & & 2 & 4 & 1 & 1 & 2 & 100 & 200 & 50 & 50 & 100 \\
\hline \multicolumn{7}{|c|}{ Demanda total por equipamento (D) } & 150 & 350 & 175 & 100 & 150 \\
\hline \multicolumn{7}{|c|}{ Coeficiente do IROG- ITO } & 0.83 & 0.73 & 0.64 & 0.76 & 0.44 \\
\hline \multicolumn{7}{|c|}{ Coeficiente do IROG- IPO } & 0.64 & 0.78 & 0.59 & 0.47 & 0.82 \\
\hline \multicolumn{7}{|c|}{ Coeficiente do IROG- IPA } & 0.83 & 0.89 & 0.98 & 0.92 & 0.89 \\
\hline \multicolumn{7}{|c|}{ Índice de Rendimento Operacional Global (IROG) } & 0.44 & 0.51 & 0.37 & 0.33 & 0.32 \\
\hline \multicolumn{7}{|c|}{ Novo Coeficiente do IROG- IPAC } & 0.83 & \multicolumn{4}{|c|}{0.80} \\
\hline \multicolumn{7}{|c|}{ Novo IROG Corrigido do sistema } & 0.44 & \multicolumn{4}{|c|}{$\overline{0.41}$} \\
\hline \multicolumn{7}{|c|}{ Capacidade nominal do equipamento (C) } & 500 & 500 & 500 & 500 & 500 \\
\hline \multicolumn{7}{|c|}{ Nova Capacidade Real do equipamento (C x IROG) } & 220 & 203 & 185 & 164 & 161 \\
\hline \multicolumn{7}{|c|}{ Nova Diferença temporal ((C x IROG)-D) } & 70 & -147 & 10 & 64 & 11 \\
\hline \multicolumn{7}{|c|}{ Quantidade de produtos não atendidos } & & -21 & & & \\
\hline
\end{tabular}

Fonte: elaborado pelos autores (2012).

A partir da análise da aplicação do modelo verificou-se que calculando o IROG Corrigido do sistema, a quantidade teórica de produtos não entregues aumentou consideravelmente em relação ao cenário inicial que desconsiderava o desempenho de qualidade após o gargalo. Esse resultado revela a distorção existente entre a capacidade real teórica do sistema e a abordagem tradicional proposta por Nakajima (1988) que não aborda a visão sistêmica do fluxo e as ocorrências que ocorrem após a restrição do sistema e impactam diretamente no seu desempenho global. Um ponto central de discussão reside no fato de que a quantidade de produtos rejeitados após o gargalo contribui para definir os níveis de capacidade reais a partir de uma determinada demanda considerada. Nesse sentido, pode-se dizer que o cálculo correto do indicador de qualidade (IPAC) é fundamental para o dimensionamento da capacidade do sistema a partir do novo índice proposto nessa pesquisa, o IROGC. O modelo, por sua vez, revelou que o novo índice do IROGC parece apresentar maior robustez na análise e gerenciamento da capacidade em sistemas de manufatura, em comparação ao índice (IROG) inicialmente apresentado pro Nakajima (1988). Tal contribuição só foi possível se ser gerada nesse artigo pela visão global do sistema produtivo oferecida pela TOC. É visível que a discussão e os achados do modelo proposto nesse artigo estão longe 
de esgotar a discussão sobre o dimensionamento da capacidade na manufatura. Entretanto, acredita-se que essa pesquisa, dentre outras contribuições, além de apresentar um modelo genérico estruturado em passos lógicos a serem usados para o gerenciamento da capacidade na gestão da manufatura, também acrescenta indicadores novos no âmbito da literatura sobre capacidade produtiva.

\section{CONSIDERAÇÕES FINAIS E TRABALHOS FUTUROS}

O presente artigo apresentou um modelo de gerenciamento da capacidade em sistemas produtivos integrando os conceitos da Teoria das Restrições com uma abordagem para o uso do IROG do TPM diferente da que consta na tradicional literatura. $O$ estudo apresentou quais indicadores de capacidade devem ser considerados e como medi-los para mensurar a real capacidade de sistemas de manufatura. $O$ presente estudo também sugeriu um modelo dividido em etapas, as quais cada etapa é pré-requisito para as demais, para dimensionar a real capacidade de um sistema produtivo frente a uma determinada relação entre capacidade e demanda. Nesse sentido, acredita-se que estudo atendeu ao primeiro objetivo proposto nessa pesquisa ao explicitar a real capacidade de um sistema produtivo, assim como atendeu ao segundo objetivo proposto ao apresentar novos indicadores (IPAC e IROGC) que devem ser considerados, bem como a maneira de calculá-los.

O artigo também explicitou o impacto na relação capacidade versus demanda existente entre a metodologia tradicional do TPM em duas perspectivas. A primeira discutiu o impacto na medição da capacidade produtiva ao se implementar o IROG apenas no gargalo. Já a segunda perspectiva, foi discutida na medida em que o modelo proposto contempla as não conformidades de qualidade que ocorrem após 0 gargalo dentro do fluxo produtivo. Ao avançar na discussão, o artigo também evidenciou o impacto ao considerar as não conformidades que ocorrem após o gargalo do fluxo produtivo em combinação com a importância do cálculo correto do indicador IPA do tradicional método de cálculo do IROG.

O modelo proposto é então apresentado como uma abordagem que não apenas contribui com o processo de tomada de decisão sobre os níveis de 
capacidade em sistemas produtivos, como também possibilita 0 melhor gerenciamento dos recursos e suas capacidades, da programação da produção no chão de fábrica e do atendimento às demandas programadas em ambientes da manufatura. Como extensão natural dessa pesquisa, os autores sugerem a aplicação de estudos de casos reais em empresas de diferentes segmentos de atuação, a fim de avaliar a abrangência de aplicação do modelo proposto, bem como a análise de outros elementos que possam ser inseridos no modelo proposto.

Por fim, os autores do presente trabalho agradecem aos revisores do artigo pelas relevantes contribuições e considerações realizadas durante o processo de avaliação.

\section{REFERÊNCIAS}

INMAN, R. A.; SALE, M. L.; GREEN JR., K. W. Analysis of the relationships among TOC use, TOC outcomes, and organizational performance. International Journal of Operations \& Production Management, v. 29, n. 4, p.341 - 356, 2009.

ANTUNES JR., J.A.V Em direção a uma teoria geral do processo na administração da produção: uma discussão sobre a possibilidade de unificação da teoria das restrições e a teoria que sustenta a construção dos sistemas de produção com estoque zero. Tese (Doutorado em Administração de Empresas)-Programa de Pós-Graduação em Administração da UFRGS, Porto Alegre, RS, 1998.

ANTUNES, Junico; ALVAREZ, Roberto; KLIPPEL, Marcelo; BORTOLOTTO, Pedro; DE PELLEGRIN, Ivan. Sistemas de produção: sistemas e práticas para projeto e gestão da produção enxuta. Porto Alegre: Bookman, 2008.

BRAGLIA, M.; FROSOLINI, M.; ZAMMORI, F. Overall equipment effectiveness of a manufacturing line (OEEML): An integrated approach to assess systems

performance. Journal of Manufacturing Technology Management, v. 20, n. 1, p. 8 $-29,2008$.

CHAKRAVORTY, S. S.; BRIAN ATWATER, J. Bottleneck management: theory and practice. Production, Planning \& Control, v. 17, n. 5, p. 441-447, 2006.

COX, J; SPENCER, M. Manual da teoria das restrições. Porto Alegre: Bookman, 2002.

DETTMER, H. W. Beyond Lean manufacturing: combining lean and the theory of constraints for higher performance. Port Angeles, US. 2001.

GOLDRATT, E M. \& COX, J.F. A meta. 1. ed. São Paulo SP: Nobel, 1984. 
GOLDRATT, E. M. A síndrome do palheiro: garimpando informações num oceano de dados. São Paulo: Editora Educator, 1996.

GOLDRATT, E. M. Standing on the shoulders of giants production concepts versus production applications: the Hitachi tool engineering example. Revista Gestão \& Produção, v. 16, n. 3, p. 333-343, 2009.

GUPTA,C. M. ; BOYD, H.L. Theory of constraints: a theory for operations management. International Journal of Operations \& Production Management, v.28, n.10, p. $991-1012,2008$.

HAYES, R. PISANO, G.; UPTON, D.; WHELLWRIGHT, S. Produção, estratégia e tecnologia: em busca da vantagem competitiva. Porto Alegre: Bookman, 2008.

HOPP, W. \& M. L. SPEARMAN. Factory Physics. Boston: Irwin, 2001.

JEONG,K-Y. ; PHILLIPS, D.T. Operational efficiency and effectiveness measurement. International Journal of Operation \& Production Management, v. 21, p. 1404-1416, 2001.

IFANDOUDAS, P. ; CHAPMAN, R. A practical approach to achieving Agility- a theory of constraints perspective. Production Planning and Control, v.8, n. 20, p. 691702, 2009.

MABIN, V. J.; BALDERSTONE, S. J. The performance of the theory of constraints methodology: analysis and discussion of successful TOC applications. International Journal of Operations \& Production Management, v. 23, n. 6, p. 568-595, 2003.

MATHUR, A; DANGAYACH, G.S.; MITTAL, M.L.; SHARMA, M. K. Performance measurement in automated manufacturing. Measuring Business Excellence, v. 15 n. 1, p. $77-91,2011$.

MUTHIAH, K.M.N.;HUANG, S.H. Overall throughput effectiveness (OTE) metric for factory-level performance monitoring and bottleneck detection, International Journal of Production Research, v. 45, n. 20, p. 4753-69, 2007.

MUTHIAH, K.M.N., HUANG, S.H.; MAHADEVAN, S. Automating factory performance diagnostics using overall throughput effectiveness (OTE) metric, International Journal of Advanced Manufacturing Technology, v. 36, n. 7/8, p. 811-24, 2008.

NAKAJIMA, S. Introduction to TPM: total productive maintenance. Cambridge, MA: Productivity Press, 1988.

RAHMAN, S. Theory of constraints: A review of the philosophy and its applications, International Journal of Operations \& Production Management, v. 18, n. 4, p. $336-355,1998$. 
ROSE, E.; ODOM, R.; DUMBAR, R.; HINCHMAN, J. How TOC \& TPM work together to build the quality toolbox of SDWTs. In: Electronics manufacturing technology symposium, manufacturing technologies - present and future, Seventeenth IEEE/CPMT International, Austin, TX , USA, 56-59, 1995.

SCOTT, D. ; PISA, R. Can overall factory effectiveness prolong moore's law? Solid StateTechnology, v. 41, n. 3, p. 75-82, 1998.

UMBLE, M.M. \& SRIKANTH, M.L. Synchronous Manufacturing: principles for world class excellence. Cincinnati, South-Western, 1995.

UMBLE, M. M; UMBLE, E. J. Drum-buffer-rope for Lower inventory. Industrial Management, p. 24-33, Sep./Oct. 1999.

WATSON, K.J. ; PATTI, A. A comparison of JIT and TOC buffering philosophies on system performance with unplanned machine downtime, International Journal of Production Research, v. 46, n. 7, p. 1869-85. 2008.

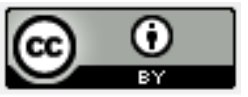

Artigo recebido em 14/04/2011 e aceito para publicação em 07/08/2012. 